\title{
Microfluidic measurement of cell motility in response to applied non-homogeneous DC electric fields
}

\author{
Marisa Rio ${ }^{1}$, Sharanya Bola ${ }^{2}$, Richard H. W. Funk ${ }^{2}$, and Gerald Gerlach ${ }^{1}$ \\ ${ }^{1}$ Solid-State Electronics Laboratory, Technische Universität Dresden, 01069 Dresden, Germany \\ ${ }^{2}$ Department of Anatomy, Technische Universität Dresden, 01307 Dresden, Germany \\ Correspondence to: Marisa Rio (marisa.rio@tu-dresden.de)
}

Received: 5 February 2016 - Revised: 8 June 2016 - Accepted: 16 June 2016 - Published: 12 July 2016

\begin{abstract}
Endogenous electric fields (EFs) play an important role in many biological processes. In order to gain an insight into these biological phenomena, externally applied electric fields are used to study cellular responses. In this work, we report the construction and fabrication of a direct current (DC)-electrically stimulated microfluidic biochip and its validation with murine photoreceptor-derived $661 \mathrm{~W}$ cells. The presented device has the particularity of offering a non-homogeneous EF environment that best resembles the endogenous electric fields in vitro. The fabrication process is relatively easy, namely by photolithography and soft lithography techniques and, furthermore, it enables live-cell imaging under an inverted microscope. First experimental results reveal cathodal directional cell migration upon applied DC EFs. In summary, the microfluidic biochip has proven biocompatibility and suitability for cellular electrotaxis experiments in non-homogeneous DC electric fields.
\end{abstract}

\section{Introduction}

Directed cell migration is essential in a variety of biological processes such as wound healing, cancer metastasis, regeneration and immune responses (Condeelis et al., 1992). There are diverse external cues like chemokines, cell-cell contacts, growth factors and the extracellular matrix environment that regulate cell migration (Entschladen and Zänker, 2010). In addition to these chemical and mechanical stimuli are the less well-recognized endogenous electric fields (EFs). Nevertheless, this electrical stimulus has been shown to play an important role in many cell biological phenomena, ranging from cell adhesion, migration, embryonic and tissue development to wound healing (Levin, 2003; Nuccitelli, 2003; Robinson and Messerli, 2003; McCaig et al., 2005; Funk et al., 2009; Messerli and Graham, 2011; Funk, 2015). Studies suggest that a large majority of the motile cells are electrically sensitive (Mycielska and Djamgoz, 2004; McCaig et al., 2005; Lin et al., 2008). Upon externally applied electric fields within physiological strength, cell-directional migration towards the anode or the cathode can be induced in a phenomenon designated electrotaxis. The direction of migration varies among cell types. Interestingly, most cells types migrate towards the cathode, with only a few migrating towards the anode (Nuccitelli, 2003).

The mechanisms driving the cells under EFs are still not very well understood. Therefore, there is a need for a system which represents an in vivo electrical environment for elucidating the EF-directed cellular mechanisms.

To date, the majority of electrotaxis devices have had only minor changes since first introduced over 30 years ago (Cooper and Keller, 1984; Erickson and Nuccitelli, 1984). Although using direct current (DC) supplies, the experiments are generally performed in unidirectional, homogeneous EFs and, consequently, cells experience uniform EF strengths. However, the endogenous electric stimulus tends to be nonhomogeneous in nature.

Herein, we describe the construction and fabrication of a microfluidic biochip, which allows the measurement of cell motility in response to non-homogeneous DC electric fields.

\section{Device fabrication}

This section describes the fabrication process of the microfluidic biochip used to study cellular electrotactic be- 

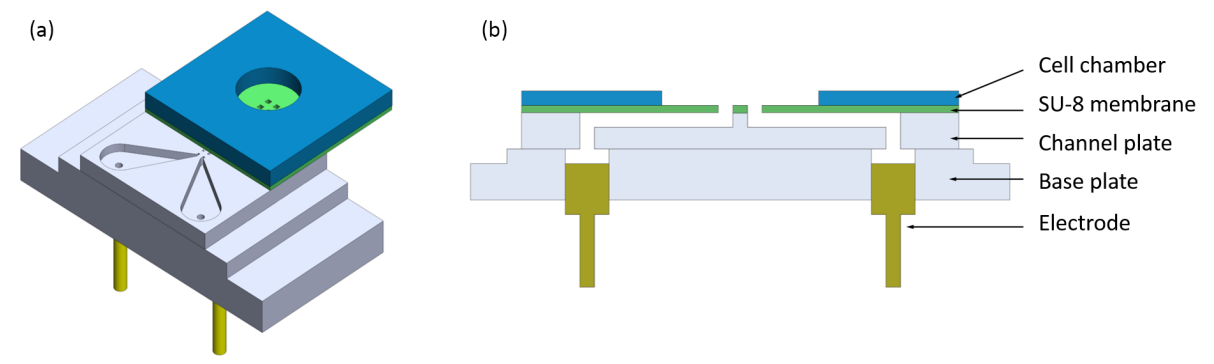

Figure 1. Schematic representation of the microfluidic biochip: (a) device assembly; (b) schematic side view.

haviour in non-homogeneous EFs. A schematic representation of the device is presented in Fig. 1. The microfluidic biochip is composed of three main components: the channel plate assembly made of polydimethylsiloxane (PDMS) on a polycarbonate (PC) base plate, the top plate which consists of an SU-8 membrane and a cell culture chamber, and lastly, the $\mathrm{Ag} / \mathrm{AgCl}$ electrodes as electrical connections to the electrolyte-filled channels. The PC base plate houses the $\mathrm{Ag} / \mathrm{AgCl}$ electrodes (World Precision Instruments, Berlin, Germany), each one connected to one end of a fluidic channel. The electrodes are encased in a $15 \mathrm{~mm}$ polyether ether ketone (PEEK) housing filled with $2 \%$ agar solution and screwed to the bottom of the base plate so they are in direct contact with the electrolyte that fills the channels. Moreover, a SU-8 free-standing membrane seals the fluidic channels, leaving only the central electrode openings connecting each electrode to the central well by its $25 \mu \mathrm{m}^{2}$ electrode openings. The set-up is finalized by a PDMS cell chamber for cell stimulation. This area is located in the centre of the chip, so that the channels are extended radially outwards. Since a low electrode resistance is desired, the electrode channels are kept as wide as possible. Furthermore, to avoid electrical interference due to leakage currents between the electrodes, the distance between the channels was maximized so that it decreases towards the centre of the chip. The nonhomogeneity of the field is achieved by using four electrodes. The four electrodes will interfere with each other, giving rise to non-linear electric field complex patterns, as demonstrated in Fig. 6a.

The microfluidic biochip fabrication encompasses three stages:

\subsection{Step 1: Fabrication of the channel plate}

At first, a SU-8 master was fabricated using standard lithography. Briefly, in order to produce channels with a thickness of $40 \mu \mathrm{m}$, SU-8 3025 (MicroChem Corp., Newton, USA) was spin-coated at $500 \mathrm{rpm}$ for $10 \mathrm{~s}$ and spread at $1000 \mathrm{rpm}$ for $30 \mathrm{~s}$. A prebaking step was carried out at $95^{\circ} \mathrm{C}$ for $30 \mathrm{~min}$. The SU-8 initiator was activated by exposure to UV for $1 \mathrm{~min}$. Subsequently, a post-exposure bake was performed at $65^{\circ} \mathrm{C}$ for $1 \mathrm{~min}$ and $95^{\circ} \mathrm{C}$ for $5 \mathrm{~min}$. Finally, the samples were developed with the SU-8 mr-Dev 600 developer (MicroChem Corp., Newton, USA), which is mainly composed of propylene glycol monomethyl ether acetate (PGMEA) and rinsed with isopropanol. Ultimately, for the purpose of prolonging the masters' lifetime and facilitating PDMS removal from the mould, the master was exposed to a (tridecafluoro1,1,2,2-tetrahydrooctyl)-1-trichlorosilane (United Chemical Technologies, USA) atmosphere for a period of $2 \mathrm{~h}$. The silanized silicon master was then used to pattern the microfluidic channels by soft lithography. Polydimethylsiloxane (PDMS) (Sylgard 184 Silicone elastomer, Dow Corning, Midland, USA) silicone base and curing agent were mixed in a $10: 1$ ratio by weight, respectively, and degassed. The mixture was subsequently injected into the MicCell platform (GesiM, Großerkmannsdorf, Germany) over the PC base plate. The curing process was done at $100^{\circ} \mathrm{C}$ for $60 \mathrm{~min}$. Once cured and cooled down, the MicCell platform was disassembled, releasing the base plate with the fluidic channel built in the PDMS polymer.

\subsection{Step 2: Fabrication of the SU-8 free-standing membrane}

The membrane is responsible for sealing the microfluidic channels and enabling the enclosed electrolyte solution to flow through the openings up to the cell culture chamber. Once the device demands for an optically transparent and biocompatible membrane were met (Nemani et al., 2013), a lift-off technique using SU-8 and standard photolithography was selected. Additionally, this method facilitates obtaining of high-resolution geometric patterns. In order to facilitate the SU-8 patterned membrane release from the substrate, an OmniCoat $^{\mathrm{TM}}$ sacrificial layer (MicroChem Corp., Westborough, USA) was spin-coated and soft-baked prior to resist deposition. The following photolithographic steps were processed as previously described, only with minor adjustments to obtain an $80 \mu \mathrm{m}$ thick photoresist layer. During the development step, the solution was gently stirred so as not to introduce additional mechanical stresses until the lift-off process was complete. 


\subsection{Step 3: Multilayer integration}

Lastly, the channel plate, the SU-8 free-standing membrane and the cell chamber were assembled. A covalent bonding of the PDMS fluidic channels to the SU-8 membrane is important for a leakage-free device. With that concern, a surface functionalization of the SU-8 free-standing membrane was performed. This was a two-step process: first the SU-8 surface was activated by $\mathrm{O}_{2}$ plasma $(100 \mathrm{~W}, 150 \mathrm{~s}$, $100 \mathrm{~W})$. Subsequently, the plasma-treated layer was soaked in $5 \%$ APTES (Sigma-Aldrich, Munich, Germany) solution and heated at $40^{\circ} \mathrm{C}$ on a hotplate for $20 \mathrm{~min}$. The APTES solution is responsible for introducing a silanized layer on the substrate, forming amine groups on the SU-8 surface. Meanwhile, the PDMS channel plate was activated by $\mathrm{O}_{2}$ plasma (same parameters). At this point, the functionalized SU-8 membrane was aligned over the fluidic channels using an optical microscope and subsequently placed over a hotplate for bonding. The optimized bonding temperature and time for our experiments were $70^{\circ} \mathrm{C}$ and $10 \mathrm{~min}$, respectively. During the bonding process, the activated amine and silanol groups condense to reduce their surface free energy. Consequently, Si-O-Si covalent bonds are formed between the SU-8 and PDMS pieces, leading to strong, irreversible bonding of the two materials (Li et al., 2012). Finally, the cell chamber was prepared by cutting a slab of PDMS $\left(22 \mathrm{~mm}^{2}\right)$ and punching it in the middle with an $8 \mathrm{~mm}$ diameter Harris, Uni-Core ${ }^{\mathrm{TM}}$ biopuncher (Ted Pella, Redding, USA). The PDMS provides a watertight reversible sealing mediated by van der Waal forces, enabling a functionalization free bonding.

The current in the DC microfluidic biochip makes its path from the $\mathrm{Ag} / \mathrm{AgCl}$ electrodes via the electrolyte medium which fills the channels to the central well chamber.

\section{Cell culture and experiment preparation}

We used an immortalized mouse retinal cell line of $661 \mathrm{~W}$, kindly provided by Muayyad Al-Ubaidi (Department of Cell Biology, University of Oklahoma Health Sciences Center, USA). The cells were cultured in Dulbecco's modified eagle medium (DMEM, Gibco, Germany) supplemented with $10 \%$ fetal calf serum (FCS, Biochrom, Germany). Cells were maintained under standard conditions $\left(37^{\circ} \mathrm{C}, 5 \% \mathrm{CO}_{2}\right)$ and allowed to grow for $24 \mathrm{~h}$ on polyethyleneterephthalate (PET) track-etched membranes ( 0.4 micron pores, BD Falcon $\left.{ }^{\mathrm{TM}}\right)$. Prior to the experiment, the device's channels were filled with a cell culture medium as an electrolyte and the $\mathrm{Ag} / \mathrm{AgCl}$ electrodes were screwed into the base plate, making sure no air bubbles were trapped at the electrode-electrolyte interface. After the incubation period, each membrane was transferred gently into the microfluidic biochip for stimulation. A glass slide was placed over the cell culture chamber closing the well. The device was subsequently transferred to the microscope with an associated incubation system and con- nected to a power supply. In all the experiments cells were exposed to a non-homogenous field of the order of $1 \mathrm{~V} \mathrm{~cm}^{-1}$ for a period of $120 \mathrm{~min}$. Membranes without electrical stimulation were taken as controls.

\section{Measurements}

\subsection{Cell viability}

Cell viability was measured using the LIVE/DEAD assay kit (Invitrogen, California, USA). After $2 \mathrm{~h}$ of EF stimulation, cells were washed with phosphate-buffered saline (PBS) containing $0.5 \mu \mathrm{m}$ of calcein AM and $6 \mu \mathrm{m}$ of ethidium homodimer-1 (EthD-1) and were incubated at $37^{\circ} \mathrm{C}$ with $5 \% \mathrm{CO}_{2}$ for $15 \mathrm{~min}$. The staining solution was removed and the samples were then viewed under an inverted microscope (Olympus IX81; Olympus, Hamburg, Germany) with 494 nm (green, calcein) and $528 \mathrm{~nm}$ (red, EthD-1) excitation filters. Images were captured using Xcellence software (Olympus, Hamburg, Germany).

\subsection{Immunofluorescence}

For the immunofluorescence staining, after $2 \mathrm{~h}$ of EF stimulation, cells were washed with PBS ( $\mathrm{pH} 7.2-7.4)$, fixed in $4 \%$ paraformaldehyde for $5 \mathrm{~min}$ at room temperature (RT), permeabilized with $0.5 \%$ Triton $\mathrm{X}-100$ for $6 \mathrm{~min}$ and subsequently blocked with $2 \%$ bovine serum albumin (BSA) for $30 \mathrm{~min}$. To detect focal contacts, cells were incubated with mouse anti-human vinculin $(1: 200$, Serotec, Martisried, Germany) at $4{ }^{\circ} \mathrm{C}$ overnight. After the incubation time, a PBS washing step followed and was later incubated with a fluorescein-isothiocyanate (FITC)-coupled goat anti-mouse antibody (1:1500, Dianova, Hamburg, Germany) along with tetramethylrhodamineisothiocyanate (TRITC)-conjugated phalloidin (1 : 300, Sigma Aldrich, Munich, Germany) at RT for $1 \mathrm{~h}$. TRITC was added to visualize actin. Finally, the nuclei were stained with 4'6diamidino-2-phenylindole dihydrochloride, DAPI (1 : 5000, Sigma-Aldrich, Munich, Germany) at RT for $5 \mathrm{~min}$. Cells mounted in DABCO were imaged under fluorescence microscope.

\subsection{Time lapse}

A real-time observation system (Fig. 2) consisting of an inverted microscope (Olympus IX81), a CCD camera (Olympus DP70), and the Xcellence imaging software together with an incubation system were used for observation of the cell migration at the microfluidic biochip. Images were recorded every $3 \mathrm{~min}$ for the duration of the experiment and a time-lapse video was created. 


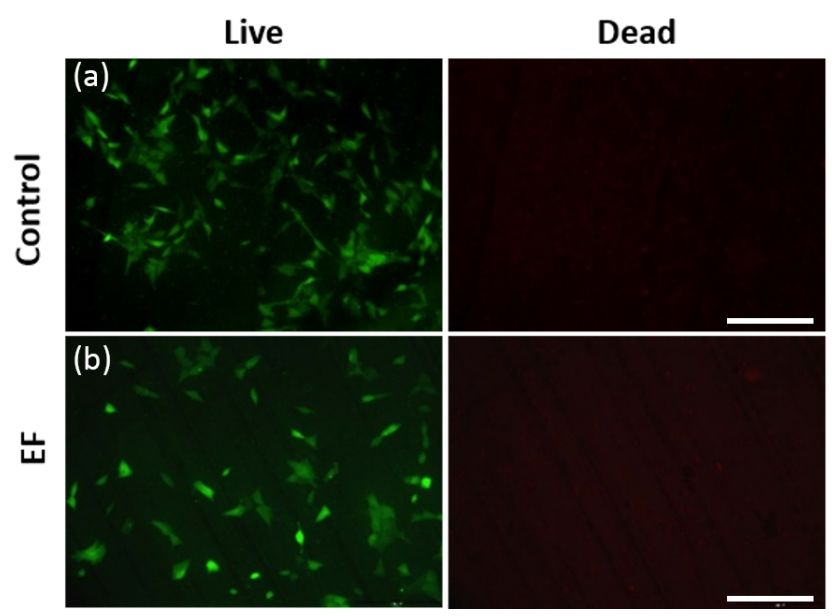

Figure 2. Illustration of the cells after $120 \mathrm{~min}$ on the device for (a) control and (b) EF stimulation. The green stain indicates viable cells, while red indicates non-viable. Scale bar $200 \mu \mathrm{m}$.

\subsection{Cell tracking and evaluation of cell migration}

For data analysis, captured images were imported into ImageJ (ImageJ $1.37 \mathrm{v}$ by W. Rusband, National Institutes of Health, Baltimore, USA). Cell movement was quantified between the frames of a temporal stack by manually tracking the centre of mass of each cell. Subsequently, the data sets of $X Y$ coordinates for each point of time were imported into the Chemotaxis and Migration Tool (v. 1.01, distributed by ibidi, Munich, Germany). After the coordinate transformation, the ibidi Chemotaxis and Migration Tool automatically sets all initial points to (0.0). This tool computed the cell migration speed and $y$-forward-migration index ( $y$-FMI) of cells and plotted the cell migration pathway. The $y$-FMI of the cell was defined as the straight-line distance along the $y$ axis between the start position and the end position of a cell divided by accumulated distance.

\section{Results and discussion}

\subsection{Cell viability assay}

The LIVE/DEAD assay enables the differentiation of metabolic active cells from damaged and dead cells (Fig. 2). Live and dead cells were checked for control as well as for stimulated samples using calcein and ethidium homodimer dyes. Cells were exposed to a non-homogenous field of the order of $1 \mathrm{~V} \mathrm{~cm}^{-1}$ for a period of $120 \mathrm{~min}$. Membranes without electrical stimulation were taken as controls. The cells stained with green are viable cells. In contrast, cells stained with red are non-viable cells. The biocompatibility of the microfluidic device was confirmed as the cells exhibit no signs of apoptosis in both control and DC stimulations.

\subsection{Electrotaxis experiment}

The performance of the microfluidic biochip for electrotaxis studies was validated by studying the $661 \mathrm{~W}$ cell line electrotactic response to non-homogenous DC EF stimulation (Fig. 3). Electric fields that produce electrotaxis are typically in the range of 0.1 to $10 \mathrm{~V} \mathrm{~cm}^{-1}$ (McCaig et al., 2009). The electric field strength at the region of interest was on average $1 \mathrm{~V} \mathrm{~cm}^{-1}$, resembling the fields observed in wound tissue (Zhao et al., 2006). This field induced a current of the order of $3 \mathrm{~mA}$, which was stable for the duration of the experiment (120 min).

The migratory behaviour of cells was quantified between frames of a temporal stack by tracking the centre of mass of at least 25 cells per experiment. Images were taken every $3 \mathrm{~min}$ for the duration of the experiment and later a timelapse video was created from the image stacks. The migration pathways were traced for both control and stimulated samples. In the absence of an applied electric field (control samples, Fig. 3a) cells had a random migration in all directions with a scattered distribution, whereas when a DC EF was applied, $661 \mathrm{~W}$ cells preferentially migrated towards the cathode, as observed in Fig. 3b. Electrotactic migration was verified to be highly directional once almost all the cells migrated cathodally. These results are in agreement with the results obtained for DC homogeneous EF stimulation (data not shown). The results confirm that the microfluidic biochip enables the application of physiologically relevant EFs and can serve as a platform for further electrotaxis experiments.

\subsection{Immunofluorescence}

Immunofluorescence labelling enables the visualization of cell biocompatibility parameters such as cell attachment and cell spreading, but also cytoskeleton organization and focal adhesion formation. The actin cytoskeleton is a highly dynamic network composed of actin polymers and a panoply of associated proteins that mediate intra- and extra-cellular movement and structural support. This dynamic structure rapidly changes shape and organization in response to stimuli.

Indeed, the migration process encompasses a cascade of intracellular signalling events that coordinate actin polarization, protrusion, cellular and membrane polarization and adhesion mechanisms (Chung et al., 2001).

In Fig. 4 are depicted images of immunofluorescence labelling for control and electrically stimulated samples. In both cases the samples were placed on the device for $120 \mathrm{~min}$ before being labelled. However, stimulated samples were electrically stimulated for the above-mentioned time frame, whilst control samples without any stimulation were taken as controls. In Fig. 4b it is noticeable that, overall, cells spread and elongate in the same direction. One can see that the actin filaments from each cell elongate in a common direction, which is perpendicular to the electric field vector present in 

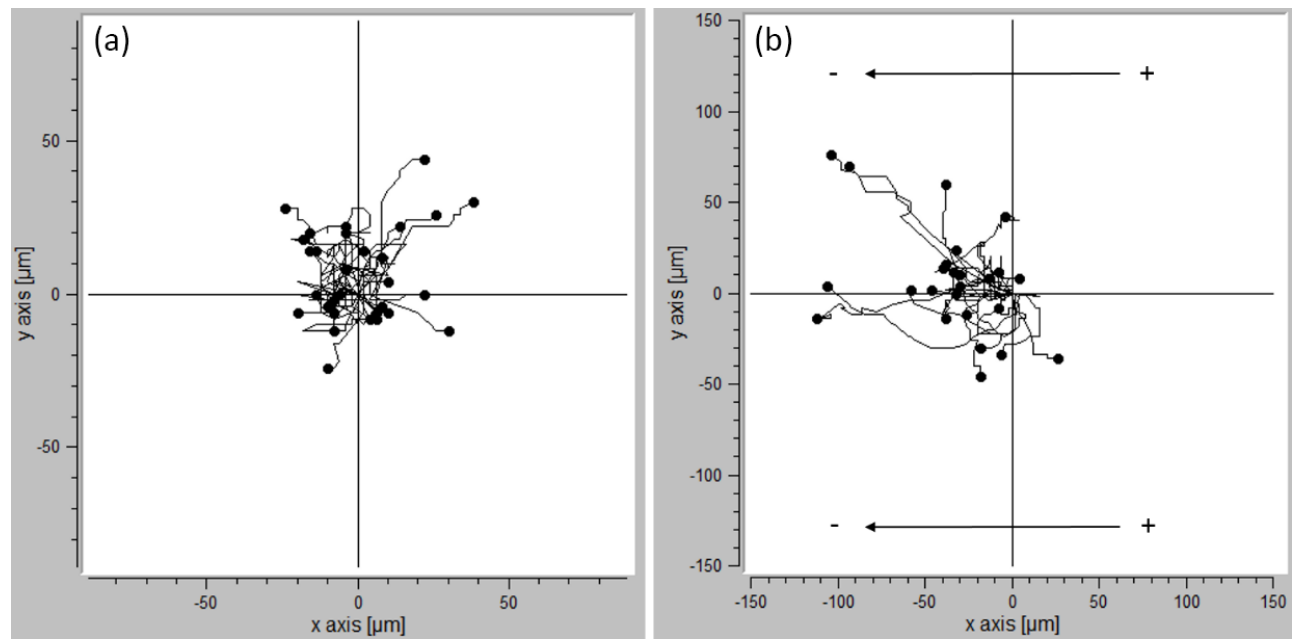

Figure 3. Directional migration of the $661 \mathrm{~W}$ cell line for (a) control and (b) applied DC EF for cell tracks of a representative experiment (120 min). The cathode and the anode are marked as - and + , respectively.

(a)
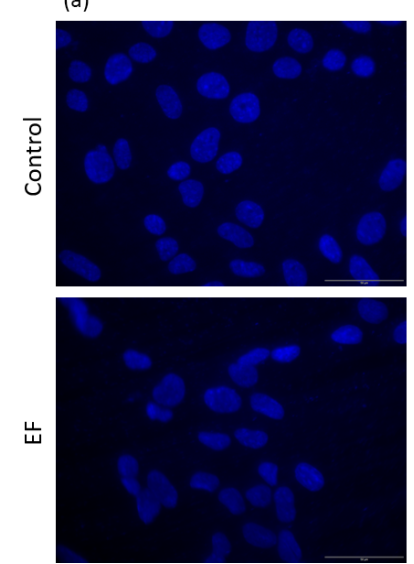

(b)
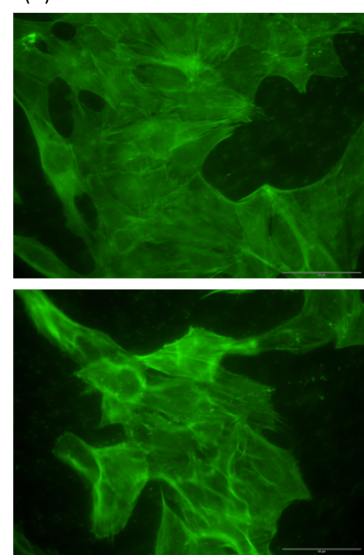

(c)
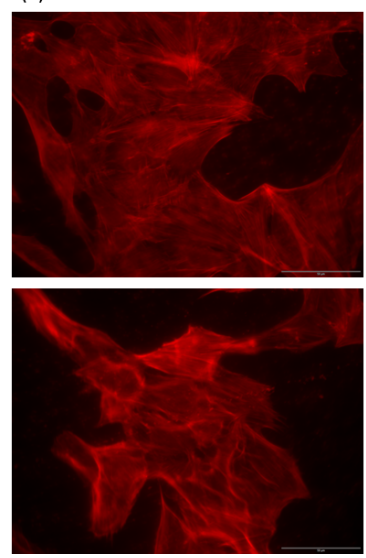

(d)

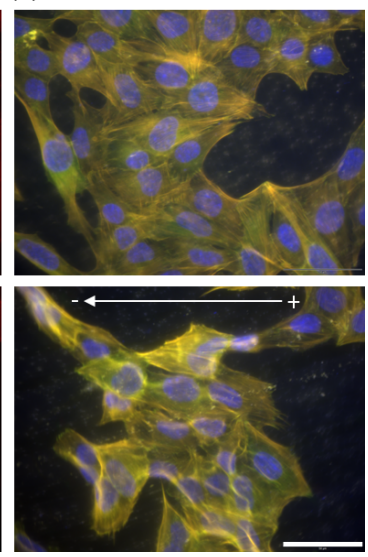

Figure 4. Morphology of $661 \mathrm{~W}$ on the PET membrane for control and after $120 \mathrm{~min}$ of applied EF. Cells were labelled for (a) nucleus, (b) vinculin, (c) filamentous actin cytoskeleton, and (d) merged image. The direction of the arrow shows the direction of the cathode and anode positions. Scale bar $50 \mu \mathrm{m}$.

the area. In contrast, the orientation of the cytoskeleton (actin filaments) and the distribution of the focal contacts (vinculin) in Fig. 4a do not seem to follow any directional cue, as cells appear to spread arbitrarily in all directions: some have actin filaments in the $x$ direction, with others in the $y$ direction. There is no external cue guiding in a specific direction, and hence cells orient and migrate randomly.

\section{Numerical simulation of electric field}

In order to estimate the electric field intensity distribution in the microfluidic biochip, a numerical simulation using commercial software (ANSYS v.16.0) run on a personal computer was performed. The steady-state electric conduction module was used in the electric field simulation. The resistivity of the medium at $37^{\circ} \mathrm{C}$ was set to $72 \Omega \mathrm{cm}$ for DMEM
(Qiu et al., 2008). Due to variations in cell growth between experiments and because the electrical properties of the cells are unknown, cells were not included in the simulations.

The finite element simulation analysed the electric field distribution inside the electrotaxis cell chamber and the electric field intensity variation in the $x$ direction. Figure 5 shows the simulation results of the electric field and current density distribution at the electrotaxis cell chamber. The arrows indicate the direction of the electric field obtained by applying 12 and $-12 \mathrm{~V}$ at the left end and at the right-hand side fluidic channels, respectively. The electric field distribution can be modified depending on the location of the anode and the cathode. A high electric field intensity is due at the centre, where the electrode openings are found (Fig. 5c), and decreases along the $x$ axis as the distance from the electrodes increases. This high intensity area englobes a $50 \mu \mathrm{m}$ radius 

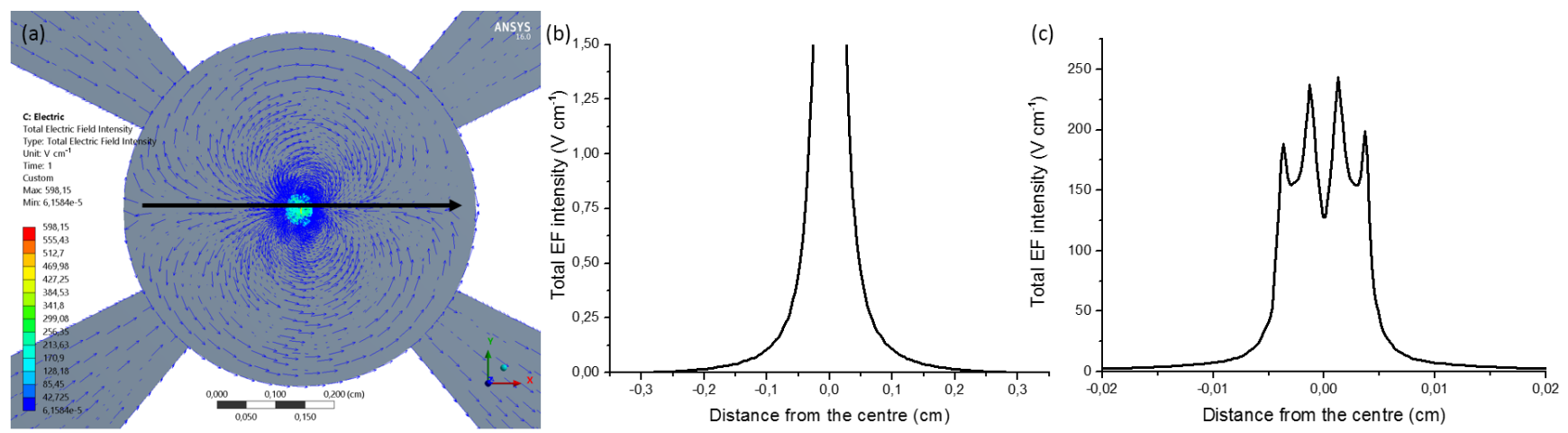

Figure 5. Simulation of the EF distribution using ANSYS 16.0. (a) EF distribution over the central cell well. Two anodes are placed at the end of the channels on the left-hand side, whilst two cathodes are at the ends of the right-hand side channels; EF profile at the region marked by the arrow over a (b) $3 \mathrm{~mm}$ radius over the channel and (c) at a $200 \mu \mathrm{m}$ radius over the electrodes where the EF intensity is at its maximum. Green represents the maximum EF intensity felt at the cell chamber, whilst blue indicates low EF. Note that the two electrode openings are the areas with higher field intensity represented by the two peaks before and the two peaks after the central point 0.0 . Scale bar $0.2 \mathrm{~cm}$.

over the centre (position marked in Fig. 5c) as (0.0) of the electrodes. From that point on the EF decreases exponentially, so that at $3 \mathrm{~mm}$ from the centre, the field is negligible.

\section{Conclusions}

In this study a new electrotaxis biochip was presented that allows the measurement of cell motility in response to nonhomogeneous DC electric fields. In contrast to the current electrotaxis devices, it has the advantage of permitting the application of non-homogenous direct current EFs that best resemble the in vivo environment near wounds. It has a compact design without the inconvenient salt bridges.

To validate the biocompatibility of the device, the cellular viability of the photoreceptor-derived $661 \mathrm{~W}$ cell line was accessed. The cells have not shown any signs of apoptosis, damage or detachment during stimulation. Furthermore, immunofluorescence staining, namely by vinculin and actin labelling, allowed the assessment of adhesion efficiency and orientation of the cytoskeleton, respectively. To demonstrate its applicability, cellular motility in the presence and absence of applied DC EFs was verified. The movement of individual cells was tracked for the duration of the experiments, confirming the EF-induced, cathodal-directed motility of the studied cell line. Simulation data confirm the application of a non-homogenous EF of physiological strength ranges.

The microfluidic biochip has proven to be biocompatible and suitable for cellular electrotaxis experiments in nonhomogeneous DC electric fields.

Further work will study the cellular response to different DC EF ranges and electrode geometries. Moreover, the intracellular and extracellular $\mathrm{pH}$ will be measured in order to give some insight into a possible silver contamination arising from the $\mathrm{Ag} / \mathrm{AgCl}$ electrodes.
Acknowledgements. This work is part of the research program of Research Training Group Nano and Bio Techniques for the Packaging of Electronic Devices financially supported by the German Research Foundation (DFG, GRK 1401).

The authors would like to thank Salvatore Girardo (Biotec, TU Dresden) for the help with the SU-8 free-standing membrane and for the fruitful discussions.

Edited by: R. Kirchner

Reviewed by: three anonymous referees

\section{References}

Chung, C. Y., Funamoto, S., and Firtel, R. A.: Signaling pathways controlling cell polarity and chemotaxis, Trends Biochem. Sci., 26, 557-566, 2001.

Condeelis, J., Jones, J., and Segall, J. E.: Chemotaxis of metastatic tumor cells: clues to mechanisms from the Dictyostelium paradigm, Cancer Metastasis Rev., 11, 55-68, 1992.

Cooper, M. S. and Keller, R. E.: Perpendicular orientation and directional migration of amphibian neural crest cells in dc electrical fields, P. Natl. Acad. Sci. USA, 81, 160-164, 1984.

Entschladen, F. and Zänker, K. S.: Cell migration: signalling and mechanisms, Transl. Res Biomed. Basel, Karger, 2, 1-6, doi:10.1159/000274472, 2010.

Erickson, C. A. and Nuccitelli, R.: Embryonic fibroblast motility and orientation can be influenced by physiological electric fields embryonic fibroblast motility and orientation, J. Cell Biol., 98, 296-307, 1984.

Funk, R. H. W.: Endogenous electric fields as guiding cue for cell migration, Front. Physiol., 6, 143, doi:10.3389/fphys.2015.00143, 2015.

Funk, R. H. W., Monsees, T., and Özkucur, N.: Electromagnetic effects - From cell biology to medicine, Prog. Histochem. Cytochem., 43, 177-264, 2009.

Levin, M.: Bioelectromagnetics in Morphogenesis, Bioelectromagnetics, 24, 295-315, 2003. 
Li, P., Lei, N., Sheadel, D. A., Xu, J., and Xue, W.: Integration of nanosensors into a sealed microchannel in an hybrid lab-on-a-chip, Sens. Actuators B, 166-167, 870-877, doi:10.1016/j.snb.2012.02.047, 2012.

Lin, F., Baldessari, F., Gyenge, C. C., Sato, T., Chambers, R. D., Santiago, J. G., and Butcher, E. C.: Lymphocyte Electrotaxis In Vitro and In Vivo, J. Immunol., 181, 2465-2471, 2008.

McCaig, C. D., Rajnicek, A. M., Song, B., and Zhao, M.: Controlling cell behavior electrically: current views and future potential, Physiol. Rev., 85, 943-978, 2005.

McCaig, C. D., Song, B., and Rajnicek, A. M.: Electrical dimensions in cell science, J. Cell Sci., 122, 4267-4276, 2009.

Messerli, M. A. and Graham, D. M: Extracellular Electrical Fields Direct Wound Healing and Regeneration, Biol Bull-US, 22, 7992, 2011.

Mycielska, M. E. and Djamgoz, M. B.: Cellular mechanisms of direct-current electric field effects: galvanotaxis and metastatic disease, J. Cell Sci., 117, 1631-1639, 2004.
Nemani, K. V., Moodie, K. L., Brennick, J. B., Su, A., and Gimi, B.: In vitro and in vivo evaluation of SU-8 biocompatibility, Mater. Sci. Eng., C 33, 4453-4459, 2013.

Nuccitelli, R.: A role for endogenous electric fields in wound healing, Curr. Top. Dev. Biol., 58, 1-26, 2003.

Qiu, Y., Liao, R., and Zhang, X.: Real-Time Monitoring Primary Cardiomyocyte Adhesion Based on Electrochemical Impedance Spectroscopy and Electrical Cell-Substrate Impedance Sensing, Anal. Chem., 80, 990-996, 2008.

Robinson, K. R. and Messerli, M. A.: Left-right, up/down: the role of endogenous electric fields as directional signals in development, repair and invasion, Bioessays, 25, 759-766, 2003.

Zhao, M., Song, B., Pu, J., Wada, T., Reid, B., Tai, G., Wang, F., Guo, A., Walczsko, P., Gu, Y., Sasaki, T., Suzuki, A., Forrester, J. V., Bourne, H. R., Devreotes, P. N., McCaig, C. D., and Penninger, J. M.: Electrical signals control wound healing through phosphatidylinositol-3-OH kinase-gamma and PTEN, Nature, 442, 457-460, doi:10.1038/nature04925, 2006. 